\title{
Prognostic Value of High CXCR4 Expression in Renal Cell Carcinoma: A System Review and Meta-Analysis
}

\author{
Yuefeng Du, Qingzhi Long, Bing Guan, and Lijun Mu \\ Department of Urology, First Affiliated Hospital of Medical School, Xian Jiaotong University, Xian, Shaanxi, China \\ Correspondence should be addressed to Yuefeng Du; mnwkdyf85323940@mail.xjtu.edu.cn
}

Received 21 June 2015; Revised 1 September 2015; Accepted 2 September 2015

Academic Editor: Stamatios Theocharis

Copyright (C) 2015 Yuefeng Du et al. This is an open access article distributed under the Creative Commons Attribution License, which permits unrestricted use, distribution, and reproduction in any medium, provided the original work is properly cited.

\begin{abstract}
Background. Recent studies have shown that CXC chemokine receptor 4 (CXCR4) is involved in the progression and metastasis of renal cell carcinoma (RCC). However, the prognostic value of CXCR4 expression in RCC remains controversial. The aim of our meta-analysis is to evaluate the prognostic value of high CXCR4 expression in RCC. Methods. Relevant studies focused on the relationship between high CXCR4 expression and the outcome of RCC were searched in PubMed and EMBASE/Cochrane Library database. Hazard ratios (HRs) of overall survival (OS) and progression-free survival (PFS) were our evaluation index. The individual and pooled HRs with 95\% confidence intervals (CIs) were analyzed. Results. A total of 1068 patients from 7 studies were included in our meta-analysis. The results suggested that high CXCR4 expression predicted a poor OS (random effect model $(\mathrm{REM}) \mathrm{HR}=2.77$, $95 \% \mathrm{CI}=1.80-4.27)$ and PFS (REM HR $=4.83,95 \% \mathrm{CI}=2.30-10.15)$ for RCC patients. Conclusion. The results of meta-analysis indicated that high CXCR4 expression was correlated with worse OS and PFS for patients with RCC. However, some larger samples and well-matched studies should be designed to estimate the potential prognosis of RCC patients.
\end{abstract}

\section{Introduction}

Renal cell carcinoma (RCC), which is the fifth most common cancer worldwide, accounts for $2-3 \%$ of all malignant diseases in adults $[1,2]$. Although an increasing number of patients with small and early stage RCC are diagnosed, there are approximately $30 \%$ of patients developing to metastatic disease and usually leading to incurable disease [3, 4]. The patients with advanced stage RCC had a low two-year survival of $8 \%$ because of chemo- and radioresistance and the cytokines toxicity profile [5-7]. Once metastasis occurred, most of the patients would relapse and finally die of the disease regardless of tyrosine-kinases and mTOR-inhibitors had been used in clinic $[8,9]$. Nevertheless, it is not active for all patients and these drugs are expensive. Thus, it is very necessary to identify prognostic and predictive markers and to develop more effective systemic therapies for different patients.

CXC chemokine receptor 4 (CXCR4) is one of 19 acknowledged chemokine receptors and belongs to the family of G-protein coupled chemokine receptor [10]. Currently, CXCR4 had been proved to be overexpressed in over 20 human malignancies, such as thyroid cancer, breast cancer, pancreatic cancer, prostate cancer, and kidney cancer [11, 12]. The results of some studies suggested that overexpression of CXCR4 which was detected in resected primary tumor tissues was associated with distal metastasis and poor prognosis $[13,14]$. Wang et al. and Zhao et al. had demonstrated that high expression of CXCR4 was strongly related to poor survival of patients with metastatic RCC $[15,16]$. Some other studies also recommended CXCR4 plus other chemokine receptors as the new biomarkers for prognosis of patients with RCC $[17,18]$. However, the prognosis value of CXCR4 is controversial because of insufficient samples and limited studies. So, we performed this meta-analysis to systematically and comprehensively evaluate the prognosis value of CXCR4 in outcome of patients with RCC.

\section{Materials and Methods}

2.1. Study Selection. Two independent authors comprehensively searched the PubMed and EMBASE/Cochrane Library for relevant articles published up to June 1, 2015. The key terms included renal cell carcinoma ("renal cancer" OR 


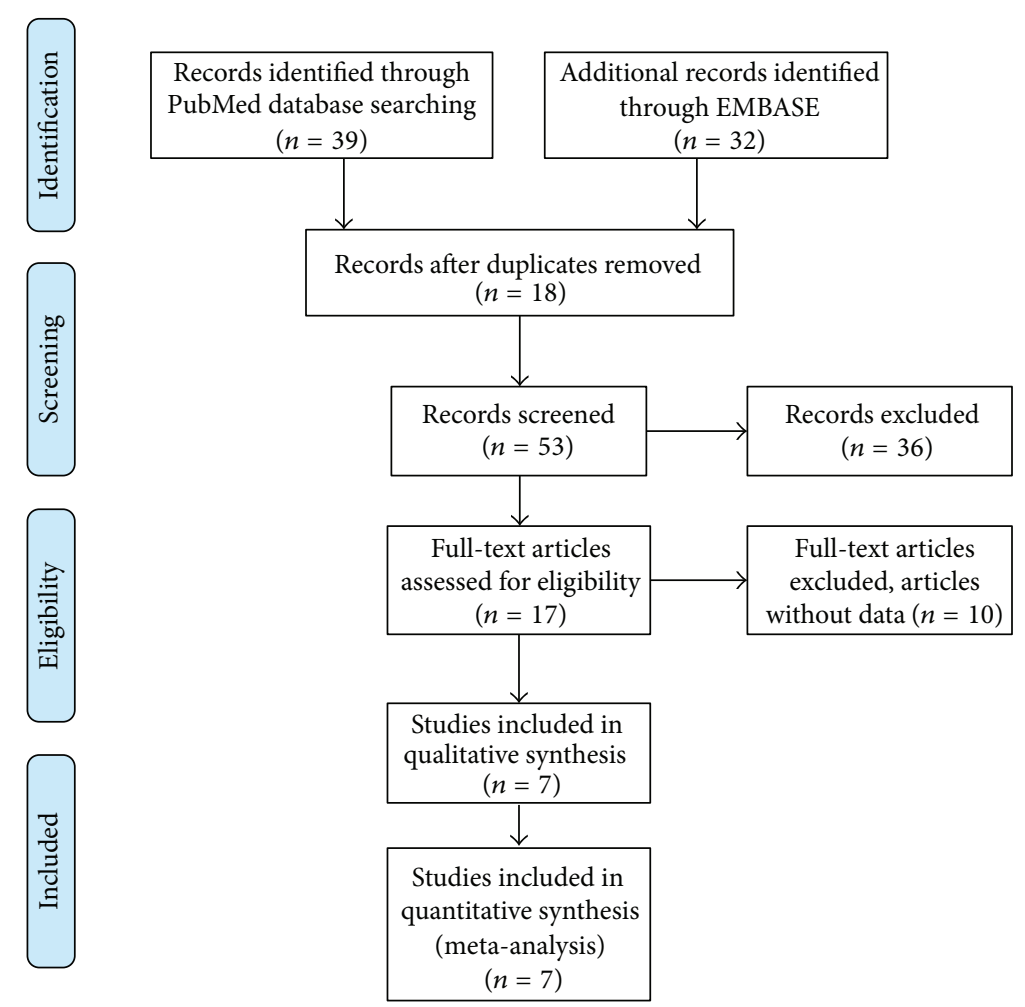

FIGURE 1: Flow diagram for articles included in this meta-analysis.

"renal cell carcinoma” OR "renal carcinoma" OR "renal tumor”), CXCR4 (“CXCR4” OR “chemokine receptor 4”), prognosis, and survival. The language of articles was limited to English. In addition, we also checked reference lists of identified studies for the other potential eligible trials. This progress was stopped when there were not additional articles.

2.2. Inclusion and Exclusion Criteria. The eligible studies included in this meta-analysis must met the following criteria to reduce the heterogeneity of articles: (1) patients with distinctive renal cell carcinoma diagnosis by pathology without the limitation of age and gender; (2) using immunohistochemistry method to detect the expression of CXCR4; (3) articles focused on the association of high CXCR4 expression and poor prognosis of patients with RCC; and (4) articles having the hazard ratios (HRs) of overall survival (OS) or progression-free survival (PFS) about CXCR4 expression and survival. The case reports, letters, and expert opinions were excluded. The exclusion criteria of studies also included (1) articles about cell lines or animals; (2) no definition of expression of CXCR4; (3) articles' lack of original data and control groups; (4) no relevant outcome data of OS or PFS; and (5) repetitive articles.

2.3. Data Abstraction and Quality Assessment. Relevant characteristics and outcome data were collected by two independent reviewers. The main characteristics of articles were listed as follows: (1) first author's name; (2) publication year; (3) country; (4) study period; and (5) median follow-up. The relevant clinical data of studies included (1) patients' number;
(2) gender (male/female); (3) age (years); (4) pathological pattern; (5) Fuhrman grade; (6) histologic origin; (7) antibody source; (8) dilution; (9) evaluation method of CXCR4 expression level; and (10) low versus high CXCR4. The HR with 95\% confidence interval (CI) was the outcome data. If an article provided the results of univariate and multivariate analyses, we chose the latter. Any disagreement was resolved by discussion. The quality of articles included in this metaanalysis was assessed by Newcastle-Ottawa Scale (NOS).

2.4. Data Analysis. A pooled analysis of HRs and 95\% CIs was used to evaluate the effect of CXCR4 expression on the survival of renal cell carcinoma in this meta-analysis. Subgroup analyses were conducted according to the ethnicity, the proportion of Fuhrman grade III-IV patients, and duration of follow-up. If the number of studies is not insufficient in subgroup analysis, then it will be listed simply. We further conducted sensitivity analyses to detect the possible reasons for heterogeneity and to evaluate the effect of each study on the overall pooled estimate. Chi square $\left(\chi^{2}\right)$ and $I^{2}$ statistics values were used for assessment of heterogeneity. There was a significant difference in survival between high and low of CXCR4 expression when $P<0.05$, except for special instructions. Fixed effect was used for meta-analysis if the value of $I^{2}>50 \%$. Otherwise, we would choose the random effect. The potential publication bias was evaluated by visually symmetry of Begg funnel plots. Moreover, Begg and Egger tests were used for the quantification of publication bias. STATA 12.0 software was used for all statistical analyses if there are no special instructions. 


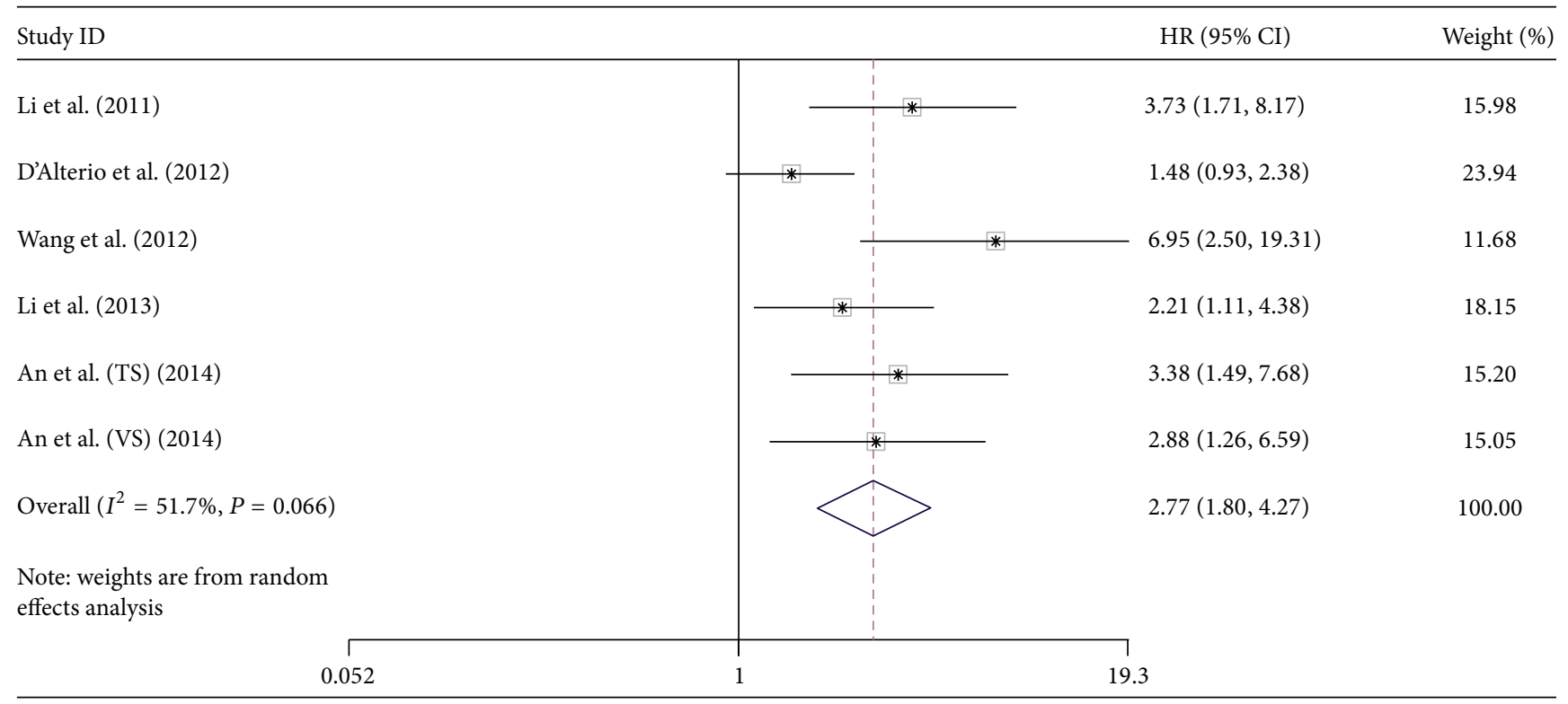

FIGURE 2: The forest plot of HRs for OS with 5 studies included in this meta-analysis.

\section{Results}

3.1. Study Characteristics. There were 7 relevant articles finally included in our meta-analysis [2,11, 17-21]. The details of selection process were shown in Figure 1. These studies were conducted in 3 countries published between 2010 and 2014 (China, France, and Italy). The total number of patients was 1068, and the sample sizes ranged from 62 to 240 patients. The median follow-up period ranged from 28.8 to 79.2 months. The main characteristics and NOS scores of articles were listed in Table 1. The baseline information and clinical data of studies included in this meta-analysis were listed in Table 2.

3.2. High CXCR4 Expression and Prognosis of RCC. There were 5 articles involved with 605 patients providing the HR of OS. The result of meta-analysis showed that high CXCR4 expression predicts a poor OS (random effect model (REM) $\mathrm{HR}=2.77,95 \% \mathrm{CI}=1.80-4.27)$ with obvious heterogeneity $\left(I^{2}=51.7 \%, P=0.066\right)$ (Figure 2). There were 6 articles involved with 843 patients investigating the HR of PFS. The results of meta-analysis showed that high CXCR4 expression also predicts a poor PFS (REM HR $=4.83,95 \% \mathrm{CI}=2.30-$ $10.15)$ with significant heterogeneity $\left(I^{2}=76.1 \%, P=0.001\right)$ (Figure 3 ). The results of sensitivity analyses showed that when the studies eliminated in turn would not change the results of pooled analyses of OS (Figure 4) and PFS (Figure 5).

3.3. Subgroup Analysis. The association between high expression and poor OS was similar in Asian patients and nonAsian patients when grouped by ethnicity and median followup period. When grouped by the proportion of Fuhrman grade III-IV patients with RCC, it seemed that there was no association between high CXCR4 expression and poor OS $(\mathrm{HR}=1.48,95 \% \mathrm{CI}=0.93-2.37)$. However, only one article was involved. The results of subgroup analyses were shown in Table 3.
3.4. Publication Bias. The interpretability of publication bias assessed by Begg and Egger tests was limited when only 7 studies were included in this meta-analysis.

\section{Discussion}

Meta-analytical technique is qualitative and quantitative tool to evaluate those subjects which are still controversial. The results of meta-analysis always were regarded as the highest level of evidence. Nowadays, people do not fully understand which factors affect the prognosis of RCC patients. Many studies had been identifying the suitable molecular biological prognostic markers for RCC. Recently, a series of studies focused on the relationship of CXCR4 expression levels and the prognosis of patients with RCC. However, these studies did not achieve consensus. This is the first meta-analysis performed to elucidate the prognosis value of high CXCR4 expression in OS and PFS of RCC patients. The results of our meta-analysis suggested that high CXCR4 expression predicted poor OS and PFS. The CXCR4 may serve as a useful prognosis marker and a therapeutic target for the RCC.

Chemokines are peptide mediators involved in normal development, immune and hematopoietic regulation, inflammation, and wound healing [10]. CXCR4 is a kind of Gprotein coupled chemokine receptor, which is always lowly or absently expressed in many normal tissues, including breast and ovary [11]. Previous studies had indicated that CXCR4 was involved in the vascularization and metastasis of cancer $[15,22]$. CXCR4 had been proved to be upregulated in many cancers, including RCC and ovarian cancer [23]. The immunochemical method showed that CXCR4 expression mainly was in cytoplasm or membrane of tumor cells of clear cell RCC (ccRCC) [24]. The precise of molecular regulation mechanism of CXCR4 in RCC remains to be determined. The protein $\mathrm{PVHL}$ coded by VHL gene may have played a key role in the mechanism of metastasis in ccRCC. The protein pVHL has the capacity to degrade hypoxia-inducible factor 
TABLE 1: Main characteristics and NOS score of each study included in meta-analysis.

\begin{tabular}{|c|c|c|c|c|c|}
\hline First author & Year & Country & Study period & $\begin{array}{l}\text { Median follow-up } \\
\text { (year) }\end{array}$ & NOS score \\
\hline An [2] & 2014 & China & 1999-2006 & $\begin{array}{l}\text { TS: } 5.2(0.6-9.7) \\
\text { VS: } 5.7(0.7-9.8)\end{array}$ & 9 \\
\hline $\mathrm{Li}[20]$ & 2013 & France & 1999-2005 & $6.6(1.0-15.3)$ & 8 \\
\hline D’Alterio [19] & 2012 & Italy & 2005-2009 & 2.4 & 8 \\
\hline Wang [21] & 2012 & China & $2002-2003$ & NA & 7 \\
\hline $\mathrm{Li}[11]$ & 2011 & China & 2001-2005 & $4.3(0.2-8.3)$ & 8 \\
\hline D’Alterio [17, 18] & 2010 & Italy & $1999-2007$ & 5.8 & 7 \\
\hline D’Alterio [17, 18] & 2010 & Italy & NA & 5.3 & 8 \\
\hline
\end{tabular}

TS: training set, VS: validation set, and NA: not available.

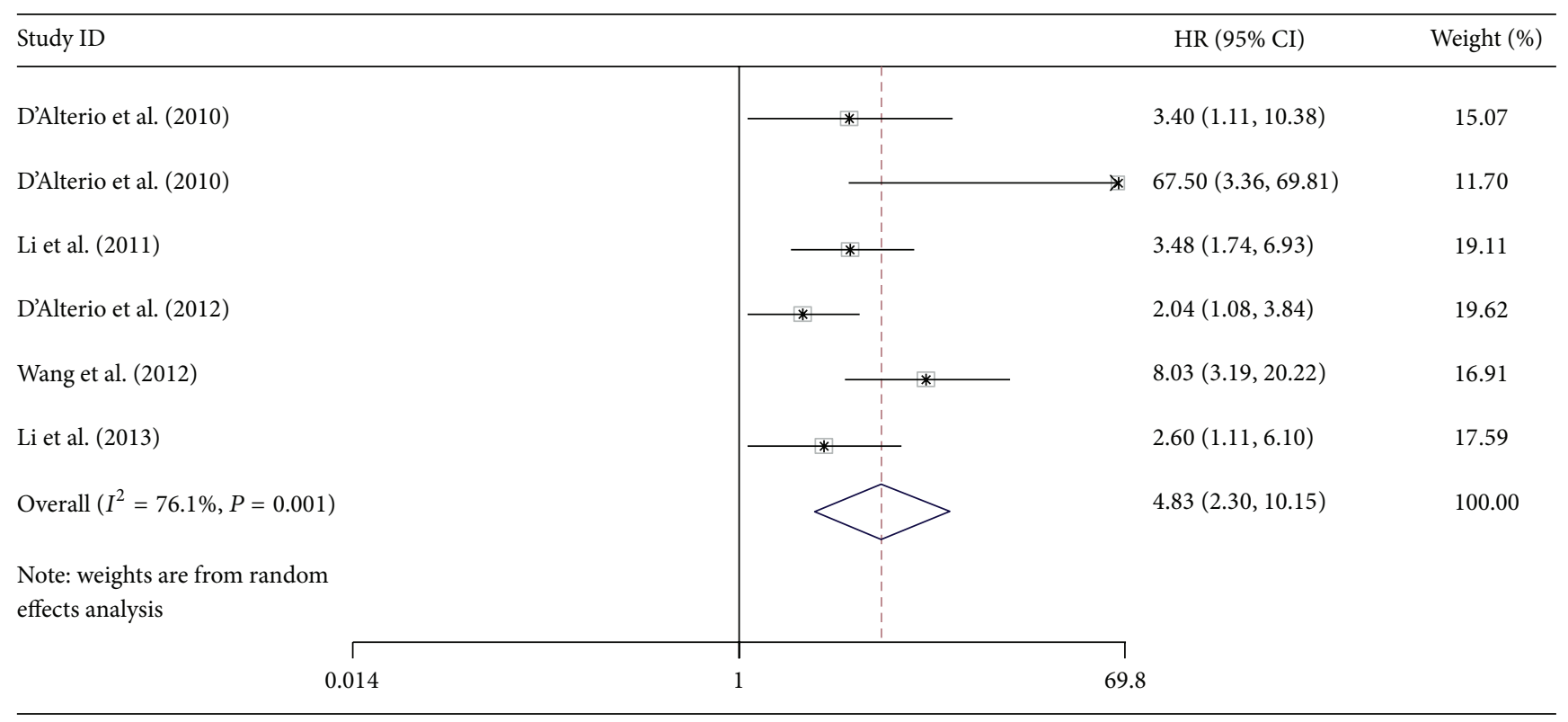

FIGURE 3: Forest plot of HRs for PFS with 6 studies included in this meta-analysis.

(HIF) under normoxic conditions [25]. This process can be suppressed under a hypoxic condition, resulting in a HIFdependent CXCR4 activation. Gahan et al. considered that CXCR4 may be crucial in controlling tumor cell adhesion via its interactions with integrin receptors [26]. Several researches also had demonstrated the importance of signaling of CXCR4 in cell-cycle regulation and apoptosis of renal cancer cells $[20,27]$. The chemokine CXCL12 (SDF-1), which is now known as an exclusive ligand of CXCR4, regulates leukocyte precursor homing to bone marrow and other sites [10]. Many studies had demonstrated that CXCR4/CXCL12 axis plays an important role in regulating metastasis of CXCR4 positive tumor cells to the organs expressing CXCL12 [11, 27]. High CXCR4 expression may affect the chemotherapy drug reaction in metastatic renal cancer. Research showed that high CXCR4 expression was correlated with a sunitinib poor response for patients with metastatic renal cancer [19]. Guo et al. also considered that patients with negative or low CXCR4 expression were more likely to obtain longer PFS [28].

A series of meta-analyses had investigated the prognosis value of CXCR4 for patients with other system tumors. Han et al. considered that CXCR4 could help predict prognosis of gastric cancer patients [29]. The results of meta-analyses from Liu et al. indicated that high CXCR4 expression was associated with poor prognosis in ovarian cancer [30]. A growing body of evidence demonstrated that CXCR4 was not the only predictor of RCC. The meta-analysis of $\mathrm{Wu}$ et al. considered that systemic inflammatory response predicted a poor survival in patients with RCC [31]. D'Alterio et al. suggested that CXCR4 combined with CXCR7 was valuable prognostic factor in RCC patients [18].

However, the results of this meta-analysis need to be interpreted cautiously due to some limitations. First, only 7 relevant articles were only involved with 1068 patients and designed in Asia and Europe. Moreover, studies in other languages were excluded except for English, so language bias may exist in our meta-analysis. Second, there was a significant heterogeneity between these studies. The clinical characteristics of patients in each study such as age, gender, and performance status would lead to bias obviously. Third, the variable histologic type and immunohistochemistry method might affect the accuracy of this meta-analysis. Further 


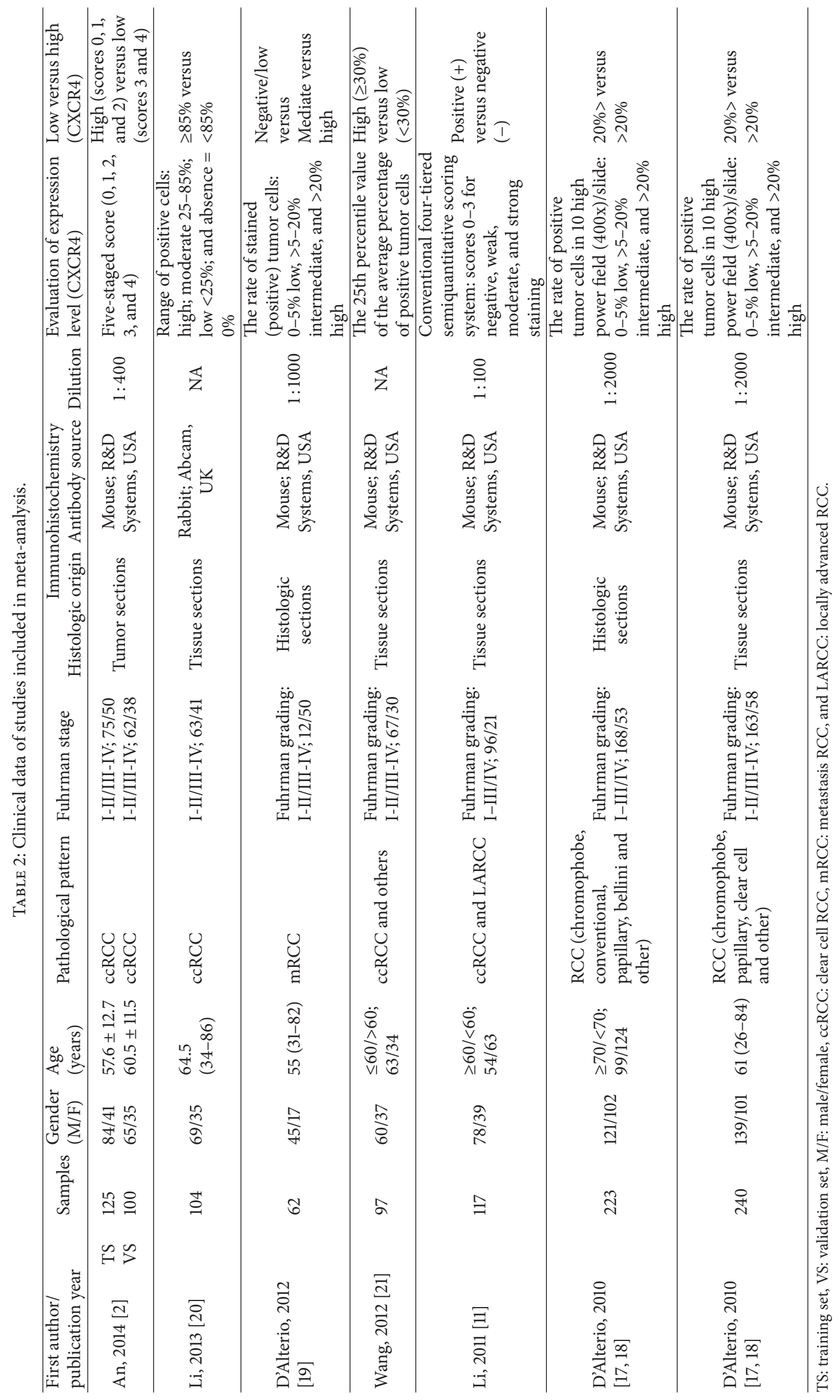


TABLE 3: Subgroup analysis based on characteristics of various studies.

\begin{tabular}{|c|c|c|c|c|c|c|c|}
\hline \multirow{2}{*}{ Variables } & \multirow{2}{*}{$\mathrm{T} / \mathrm{P}$} & \multicolumn{6}{|c|}{ Overall survival } \\
\hline & & HR (95\% CI) & $I$-squared (\%) & Model & $P-\mathrm{He}^{*}$ & $Z$ value & $P$ value \\
\hline Overall & $5 / 605$ & $2.77(1.80,4.27)$ & 51.7 & Random effect & 0.066 & 4.61 & $<0.001$ \\
\hline \multicolumn{8}{|l|}{ Ethnicity } \\
\hline Asian & $3 / 439$ & $3.78(2.47,5.78)$ & 0.0 & Fixed effect & 0.60 & 6.13 & $<0.001$ \\
\hline Non-Asian & $2 / 166$ & $1.68(1.14,2.48)$ & 0.0 & Fixed effect & 0.35 & 2.63 & 0.009 \\
\hline \multicolumn{8}{|c|}{ Fuhrman grades III-IV (\%) } \\
\hline$>70$ & $1 / 62$ & $1.48(0.93,2.37)$ & NA & NA & NA & 1.64 & 0.102 \\
\hline$\leq 70$ & $4 / 543$ & $3.26(2.27,4.67)$ & 0.0 & Fixed effect & 0.471 & 6.41 & $<0.001$ \\
\hline \multicolumn{8}{|c|}{ Median follow-up (months) } \\
\hline$>60$ & $2 / 329$ & $2.70(1.73,4.21)$ & 0.0 & Fixed effect & 0.727 & 4.39 & $<0.001$ \\
\hline$\leq 60$ & $3 / 276$ & $3.10(1.24,7.80)$ & 78.6 & Random effect & 0.009 & 2.41 & 0.016 \\
\hline
\end{tabular}

T/P: number of trials/number of patients, $P-\mathrm{He}^{*}: P$ value of heterogeneity.

Meta-analysis estimates, given named study is omitted

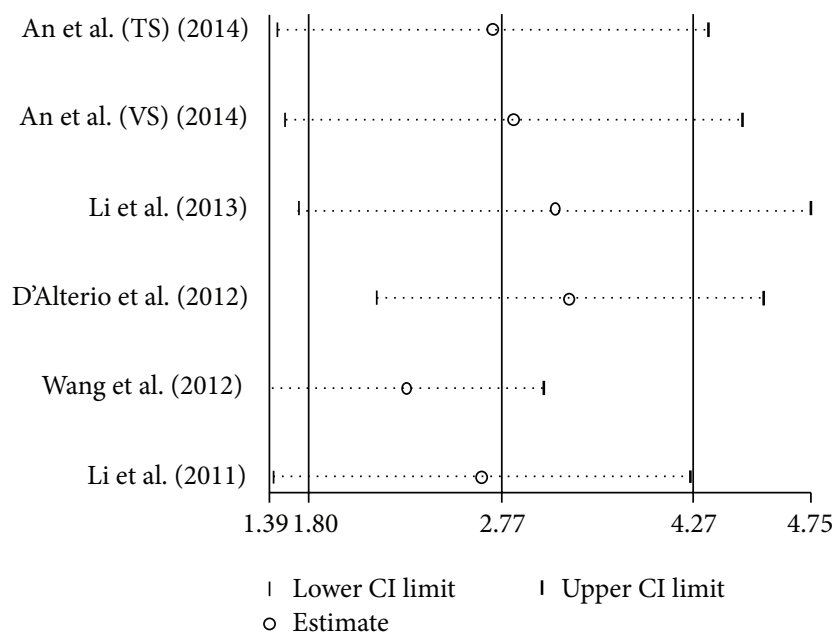

FIGURE 4: The plot of result of sensitivity analysis for OS.

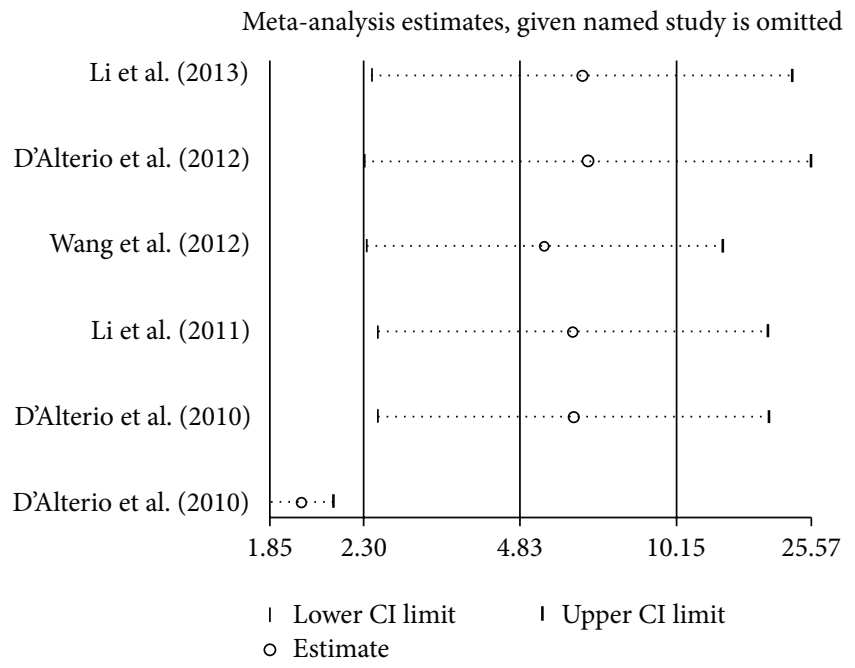

FIGURE 5: The plot of result of sensitivity analysis for PFS. researches should be conducted to investigate whether these factors would affect the results of meta-analysis.

\section{Conflict of Interests}

The authors declare that there are no other competing financial interests.

\section{Acknowledgment}

This work was supported by the National Science Foundation of China (Grant no. 81372736/H1619).

\section{References}

[1] C. E. Desantis, C. C. Lin, A. B. Mariotto et al., "Cancer treatment and survivorship statistics, 2014," CA: A Cancer Journal for Clinicians, vol. 64, no. 4, pp. 252-271, 2014.

[2] H. An, L. Xu, Y. Zhu et al., "High CXC chemokine receptor 4 expression is an adverse prognostic factor in patients with clearcell renal cell carcinoma," British Journal of Cancer, vol. 110, no. 9, pp. 2261-2268, 2014.

[3] G. D. Stewart, F. C. O'Mahony, T. Powles, A. C. P. Riddick, D. J. Harrison, and D. Faratian, "What can molecular pathology contribute to the management of renal cell carcinoma?" Nature Reviews Urology, vol. 8, no. 5, pp. 255-265, 2011.

[4] P. I. Karakiewicz, A. Briganti, F. K.-H. Chun et al., "Multiinstitutional validation of a new renal cancer-specific survival nomogram," Journal of Clinical Oncology, vol. 25, no. 11, pp. 1316-1322, 2007.

[5] J. A. Garcia and B. I. Rini, "Recent progress in the management of advanced renal cell carcinoma," CA Cancer Journal for Clinicians, vol. 57, no. 2, pp. 112-125, 2007.

[6] L. J. Costa and H. A. Drabkin, "Renal cell carcinoma: new developments in molecular biology and potential for targeted therapies," Oncologist, vol. 12, no. 12, pp. 1404-1415, 2007.

[7] W. M. Linehan, "Targeting VEGF receptors in kidney cancer," The Lancet Oncology, vol. 8, no. 11, pp. 956-957, 2007.

[8] A. Morabito, E. De Maio, M. Di Maio, N. Normanno, and F. Perrone, "Tyrosine kinase inhibitors of vascular endothelial growth factor receptors in clinical trials: current status and future directions," Oncologist, vol. 11, no. 7, pp. 753-764, 2006. 
[9] J. Brugarolas, "Renal-cell carcinoma-molecular pathways and therapies," The New England Journal of Medicine, vol. 356, no. 2, pp. 185-187, 2007.

[10] C. L. Richard and J. Blay, "CXCR4 in cancer and its regulation by PPAR $\gamma$," PPAR Research, vol. 2008, Article ID 769413, 19 pages, 2008.

[11] X. Li, Y. Huang, J. Xia et al., "Cxcr4 expression in patients with high-risk locally advanced renal cell carcinoma can independently predict increased risk of disease progression and poor overall survival," Asian Pacific Journal of Cancer Prevention, vol. 12, no. 12, pp. 3313-3318, 2011.

[12] T. Nagasawa, "CXC chemokine ligand 12 (CXCL12) and its receptor CXCR4," Journal of Molecular Medicine, vol. 92, no. 5, pp. 433-439, 2014.

[13] J. Kim, T. Mori, S. L. Chen et al., "Chemokine receptor CXCR4 expression in patients with melanoma and colorectal cancer liver metastases and the association with disease outcome," Annals of Surgery, vol. 244, no. 1, pp. 113-120, 2006.

[14] R. Maréchal, P. Demetter, N. Nagy et al., "High expression of CXCR4 may predict poor survival in resected pancreatic adenocarcinoma," British Journal of Cancer, vol. 100, no. 9, pp. 1444-1451, 2009.

[15] L. Wang, L. Wang, B. Yang et al., "Strong expression of chemokine receptor CXCR4 by renal cell carcinoma cells correlates with metastasis," Clinical and Experimental Metastasis, vol. 26, no. 8, pp. 1049-1054, 2009.

[16] F.-L. Zhao and W. Guo, "Expression of stromal derived factor1 (SDF-1) and chemokine receptor (CXCR4) in bone metastasis of renal carcinoma," Molecular Biology Reports, vol. 38, no. 2, pp. 1039-1045, 2011.

[17] C. D’Alterio, L. Cindolo, L. Portella et al., "Differential role of CD133 and CXCR4 in renal cell carcinoma," Cell Cycle, vol. 9, no. 22, pp. 4492-4500, 2010.

[18] C. D’Alterio, C. Consales, M. Polimeno et al., "Concomitant CXCR4 and CXCR7 expression predicts poor prognosis in renal cancer," Current Cancer Drug Targets, vol. 10, no. 7, pp. 772-781, 2010.

[19] C. D’Alterio, L. Portella, A. Ottaiano et al., "High CXCR4 expression correlates with sunitinib poor response in metastatic renal cancer," Current Cancer Drug Targets, vol. 12, no. 6, pp. 693-702, 2012.

[20] G. Li, G. Badin, A. Zhao et al., "Prognostic value of CXCR4 expression in patients with clear cell renal cell carcinoma," Histology and Histopathology, vol. 28, no. 9, pp. 1217-1222, 2013.

[21] L. Wang, W. Chen, L. Gao et al., "High expression of CXCR4, CXCR7 and SDF-1 predicts poor survival in renal cell carcinoma," World Journal of Surgical Oncology, vol. 10, article 212, 2012.

[22] K. Tachibana, S. Hirota, H. Iizasa et al., "The chemokine receptor CXCR4 is essential for vascularization of the gastrointestinal tract," Nature, vol. 393, no. 6685, pp. 591-594, 1998.

[23] B. Furusato, A. Mohamed, M. Uhlén, and J. S. Rhim, "CXCR4 and cancer," Pathology International, vol. 60, no. 7, pp. 497-505, 2010.

[24] T. C. Wehler, C. Graf, S. Biesterfeld et al., "Strong expression of chemokine receptor CXCR4 by renal cell carcinoma correlates with advanced disease," Journal of Oncology, vol. 2008, Article ID 626340, 2008.

[25] P. Staller, J. Sulitkova, J. Lisztwan, H. Moch, E. J. Oakeley, and W. Krek, "Chemokine receptor CXCR4 downregulated by von Hippel-Lindau tumour suppressor pVHL," Nature, vol. 425, no. 6955, pp. 307-311, 2003.
[26] J. C. Gahan, M. Gosalbez, T. Yates et al., "Chemokine and chemokine receptor expression in kidney tumors: molecular profiling of histological subtypes and association with metastasis," The Journal of Urology, vol. 187, no. 3, pp. 827-833, 2012.

[27] J. Pan, J. Mestas, M. D. Burdick et al., "Stromal derived factor-1 (SDF-1/CXCL12) and CXCR4 in renal cell carcinoma metastasis," Molecular Cancer, vol. 5, article 56, 2006.

[28] J. Guo, B. Tang, X. N. Sheng, and C. L. Cui, "Use of CXCR4 expression to predict the efficacy of sorafenib treatment in patients with metastatic renal cell carcinoma," Journal of Clinical Oncology, vol. 29, no. 7, pp. 359-364, 2011.

[29] M. Han, S. Lv, Y. Zhang et al., "The prognosis and clinicopathology of CXCR4 in gastric cancer patients: a meta-analysis," Tumor Biology, vol. 35, no. 5, pp. 4589-4597, 2014.

[30] C.-F. Liu, S.-Y. Liu, X.-Y. Min et al., "The prognostic value of CXCR4 in ovarian cancer: a meta-analysis," PLoS ONE, vol. 9, no. 3, Article ID e92629, 2014.

[31] Y. Wu, X. Fu, X. Zhu et al., "Prognostic role of systemic inflammatory response in renal cell carcinoma: a systematic review and meta-analysis," Journal of Cancer Research and Clinical Oncology, vol. 137, no. 5, pp. 887-896, 2011. 


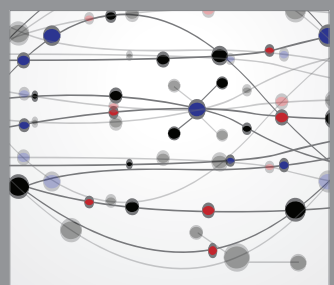

The Scientific World Journal
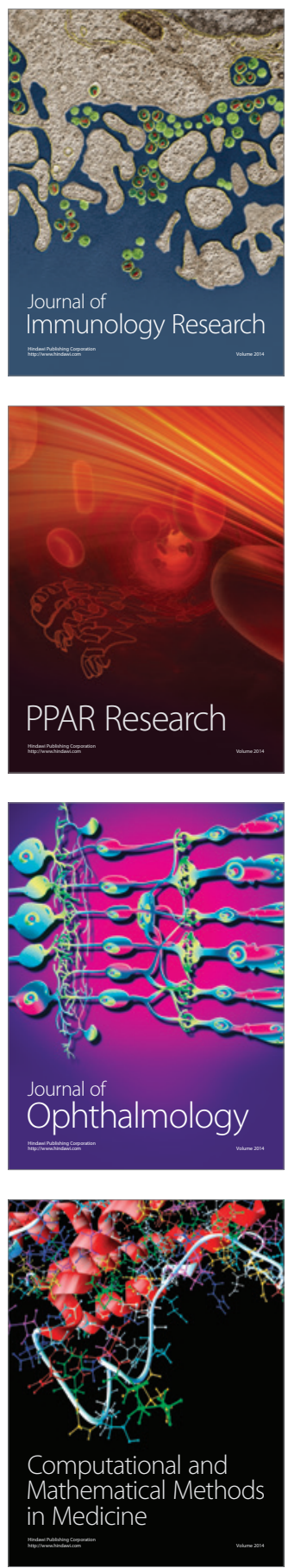

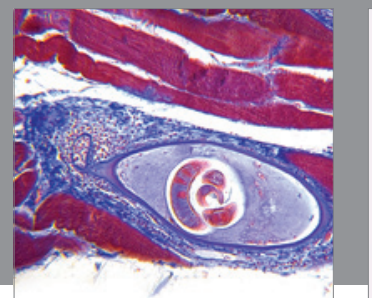

Gastroenterology

Research and Practice
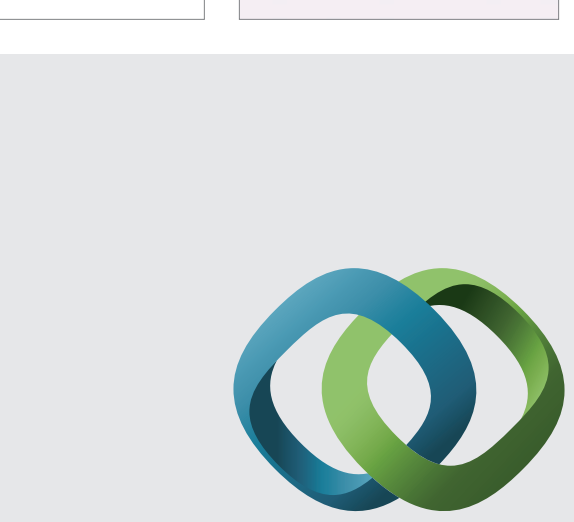

\section{Hindawi}

Submit your manuscripts at

http://www.hindawi.com
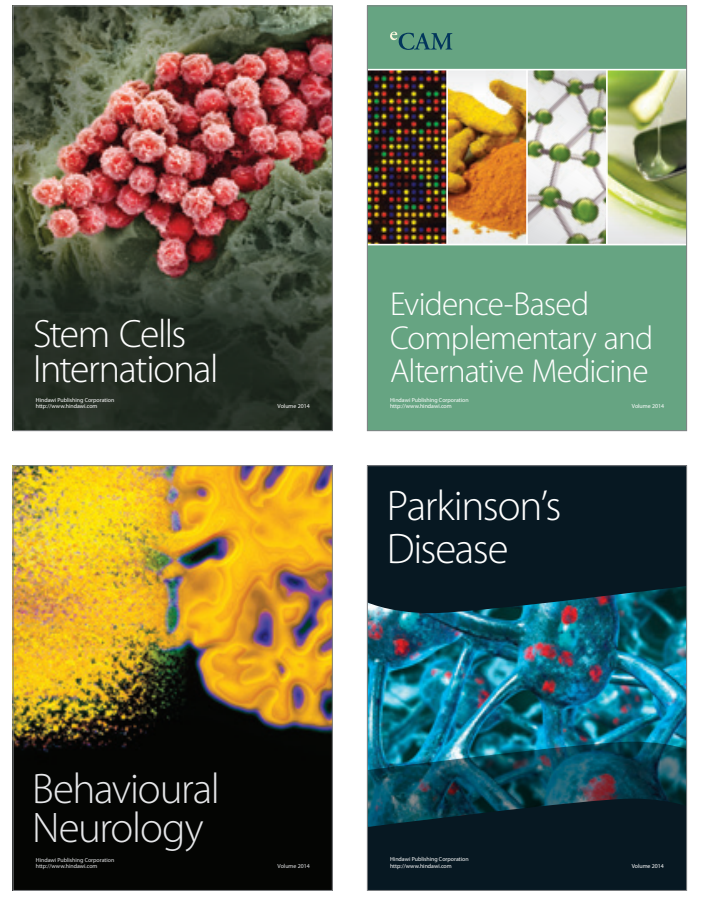
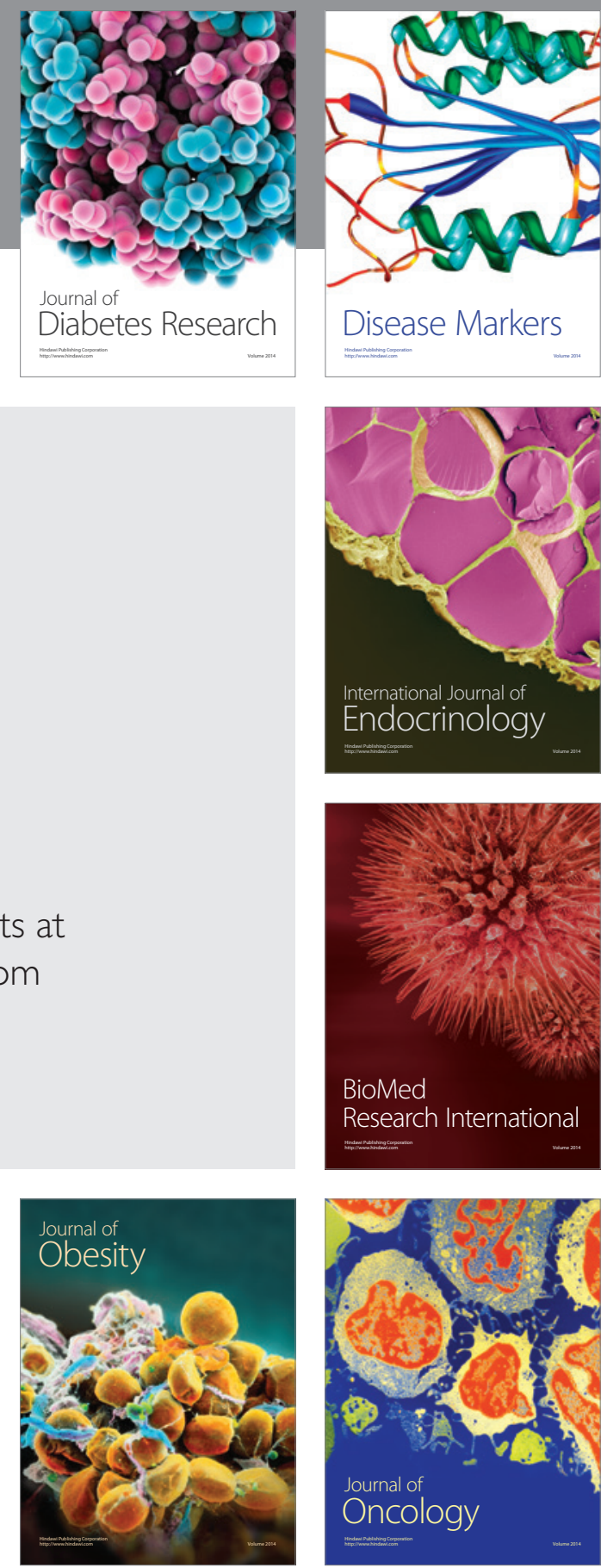

Disease Markers
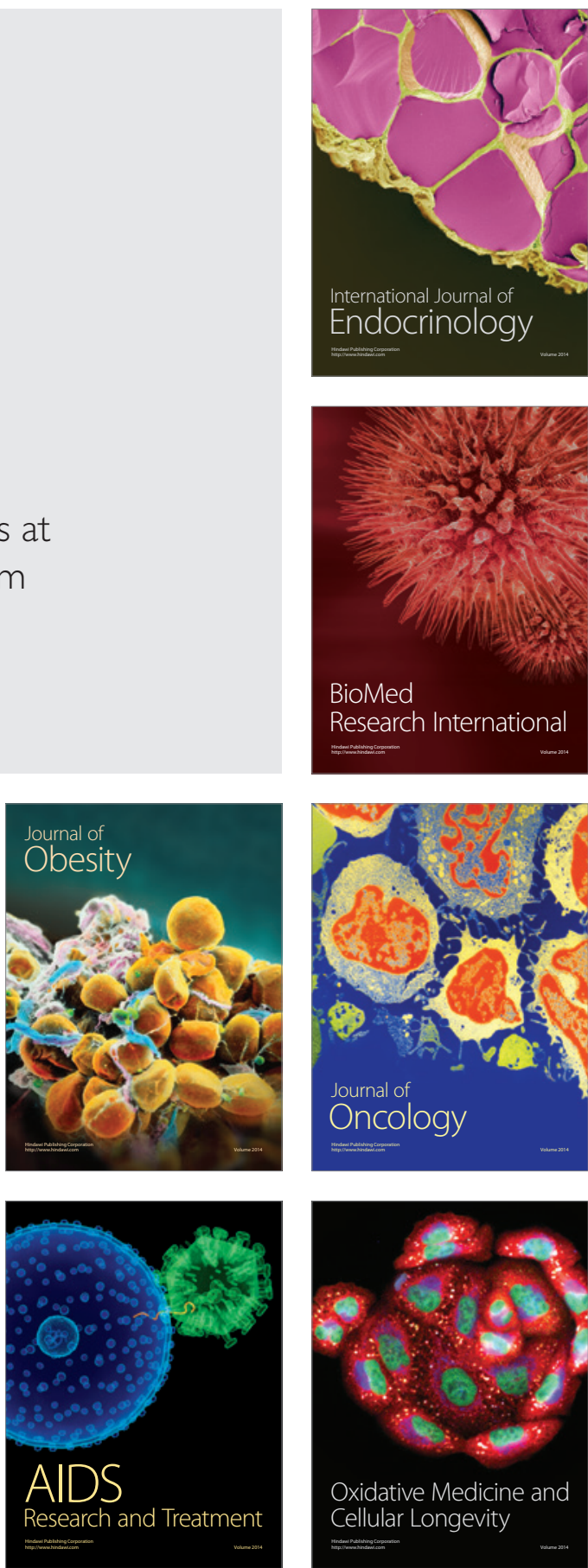